
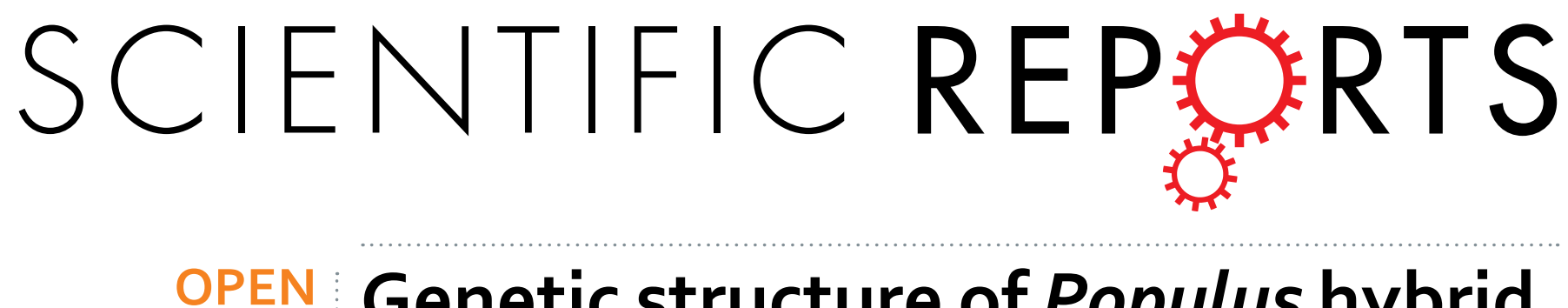

\title{
Genetic structure of Populus hybrid zone along the Irtysh River provides insight into plastid-nuclear incompatibility
}

Accepted: 25 May 2016

Published: 16 June 2016

\section{Yan-Fei Zeng ${ }^{1}$, Jian-Guo Zhang ${ }^{1,2}$, Ai-Guo Duan ${ }^{1}$ \& Bawerjan Abuduhamiti ${ }^{3}$}

In plants, the maintenance of species integrity despite hybridization has often been explained by the co-adaption of nuclear gene complexes. However, the interaction between plastid and nuclear sub-genomes has been underestimated. Here, we analyzed the genetic structure of a Populus alba and $P$. tremula hybrid zone along the Irtysh River system in the Altai region, northwest China, using both nuclear microsatellites and plastid DNA sequences. We found high interspecific differentiation, although the hybrid $P$. $\times$ canescens was prevalent. Bayesian inference classified most hybrids into F1, followed by a few back-crosses to $P$. alba, and fewer F2 hybrids and back-crosses to $P$. tremula, indicating a few introgressions but preference toward $P$. alba. When plastid haplotypes in parental species were distinct, $P . \times$ canescens carried the haplotypes of both parents, but showed significant linkage between intraspecific haplotype and nuclear genotypes at several microsatellite loci. Selection, rather than migration and assortative mating, might have contributed to such plastid-nuclear disequilibria. By removing later-generated hybrids carrying interspecific combinations of haplotype and nuclear genotypes, plastid-nuclear incompatibility has greatly limited the gene exchange between $P$. alba and $P$. tremula via backcrossing with hybrids, demonstrating a significant association between plastid haplotype and the proportion of nuclear admixture.

In nature, species often maintain their integrity in the absence of complete geographical or prezygotic isolation ${ }^{1-4}$. One of the explanations is that hybrids between species frequently show reduced fitness compared to their parents due to extrinsic or intrinsic factors and are thus quickly eliminated by selection ${ }^{1,4,5}$. Intrinsic isolation is most often attributed to the breakdown of co-adapted nuclear gene complexes (outbreeding depression) ${ }^{6-8}$. One hypothesis for such breakdown is the genetic incompatibilities described by the Bateson-Dobzhansky-Muller (BDM) model, in which substitutions that arise in different lineages cause incompatibilities when combined in a hybrid genome ${ }^{9,10}$. Sometimes, hybrids can be as fit or even fitter than their parental species, forming a new hybrid species ${ }^{2}$, whereas declines in fitness can be found in the second (or later) hybrid generations due to the greater severity of homozygous BDM incompatibilities ${ }^{11}$.

Cellular sub-genomes in the nucleus, mitochondria, and plastids communicate in complex ways and co-evolve ${ }^{12}$. Hybridization can lead to new interactions between the nuclear and cytoplasmic genomes as well as between different nuclear genomes, and this may result in cytonuclear incompatibilities (see references in Greiner et al. ${ }^{13}$ ). However, in contrast to mitochondrial-nuclear incompatibility that is of well-known evolutionary importance in animals ${ }^{12}$, plastid-nuclear interactions have not been extensively studied and hence the role of plastid-nuclear incompatibility in hybrid breakdown and speciation has been largely overlooked ${ }^{13,14}$, although interspecific plastid-nuclear incompatibilities have been widely reported ${ }^{15}$ and their implications for speciation were already recognized in the early period of formal genetics ${ }^{16}$. Recent studies on a few taxa have clearly shown that plastids establish hybridization barriers and play a major role in speciation ${ }^{17,18}$, but the occurrence of plastid-nuclear incompatibility in natural populations has been underestimated ${ }^{12,13}$.

${ }^{1}$ State Key Laboratory of Tree Genetics and Breeding, Key Laboratory of Tree Breeding and Cultivation, State Forestry Administration, Research Institute of Forestry, Chinese Academy of Forestry, Beijing, China. ${ }^{2}$ Collaborative Innovation Center of Sustainable Forestry in Southern China, Nanjing Forestry University, Nanjing, China. ${ }^{3}$ Forest Research Institute of the Altai Region, Xinjiang, China. Correspondence and requests for materials should be addressed to J.-G.Z. (email: zhangjg@caf.ac.cn) 
Hybrid zones have often been considered as "natural laboratories for evolutionary studies"19, because they can contain a wide variety of genotypes that result from many generations of recombination. First, the nuclear genetic composition of individuals in a hybrid zone reflects the intensity of reproductive isolation and gene flow ${ }^{20}$. In general, the occurrence of multiple generations of hybrids within a hybrid zone indicates a high rate of interspecific gene flow; while a scarcity of post-F1 hybrids, such as F2 individuals and back-crosses, reveals the establishment of a barrier between the parental species ${ }^{20}$. In this case, the patterns of nuclear and cytoplasmic genetic structure in hybrid zones would reflect the combined effects of selection and gene flow ${ }^{21}$. Cytonuclear disequilibrium (CND) occurs if the cytoplasmic genomes of one species combine non-randomly with the nuclear genome of the other species due to migration, assortative mating, selection, and genetic drift ${ }^{22}$. In particular, cytonuclear incompatibility can contribute to the maintenance of disequilibria and influence the extent and pattern of introgression in populations ${ }^{23}$.

Populus alba L. (white poplar) and P. tremula L. (European aspen) are ecologically and morphologically well-differentiated species ${ }^{24-26}$. $P$. alba is widely distributed in river basins over northern Africa, southern Europe, and central Asia, while the P. tremula habitat is found in the mountainous regions of the boreal and temperate deciduous parts of the Eurasian continent. The distributions of these two species overlap considerably along European river systems, and can form large mosaic hybrid zones ${ }^{24,27}$. The diploid hybrid, $P . \times$ canescens (gray poplar), is frequently found close to flood-plain forests of $P$. alba ${ }^{24,28,29}$. Genetic analyses of Populus hybrid zones in Europe have shown that, even in sympatric settings, parental species and their hybrids form three distinct ancestry groups ${ }^{27,28}$, with a strongly varied introgression from $P$. tremula into $P$. alba across marker loci ${ }^{30}$. Hybrids are genetically and phenotypically mainly intermediate between the parental species ${ }^{25,27}$. Few mature trees show backcross-like genotypes, and F1 hybrid genotypes that early studies suggested to be either very rare or absent ${ }^{27,28}$ actually prevail in $P . \times$ canescens $s^{31,32}$. Strong genomic discontinuities between hybrids and their parents, but weak reproductive isolation early in the life-cycle indicate that Populus hybrids act as strong genotypic filters ${ }^{27,28,31}$. A considerable amount of selection on some hybrid genotypes, such as backcrosses, might take place between the seedling stage and maturity ${ }^{31}$. The reduced fitness of hybrid seedlings, however, was often explained by the breakdown of co-adapted nuclear gene complexes but the plastid-nuclear interactions were overlooked. Especially, significant lineage disequilibria between the plastid variation and several nuclear loci have been detected in European hybrid zones ${ }^{33}$, suggesting non-random combination between plastid and nuclear genome in Populus. Thus, an alternative possibility is that incompatibility between the maternally-inherited plastid genome ${ }^{34}$ from one species and the biparentally-inherited nuclear genome from the other in hybrids have resulted in the "breakdown" of fitness, and finally exhibited significant linkage between the plastid genome and certain nuclear loci in the adult after selection against "incompatible" combinations. When the F1 hybrids in $P . \times$ canescens are as fit or even fitter than their parental species due to epistatic interactions within genomes ${ }^{28}$, declines in fitness can occur in the second (or later) hybrid generations due to the greater severity of incompatibilities between the plastid and homozygous nuclear genomes from different parental species ${ }^{12}$.

A natural hybrid zone between $P$. alba and P. tremula also occurs along the Irtysh River system in the Altai region of Northern Xinjiang, China ${ }^{35}$. Within this zone, the $P$. alba population is distributed in the lowland flood-plains of the Irtysh River, P. tremula populations are found in nearby mountainous regions, and their hybrids, $P . \times$ canescens, are prevalent along the tributaries of the Irtysh River, such as the Haba, Buerjin, and Buliezi Rivers. In this study, we described the genetic structure of individuals in the P. alba and P. tremula hybrid zone along the Irtysh River system, using both nuclear microsatellite and plastid DNA (i.e. chloroplast DNA, cpDNA) trnL-trnF sequences to test whether plastid-nuclear incompatibility contributes to maintaining the integrity of Populus species in the face of gene flow. More specifically, we aimed to answer the following questions, 1) Do P. alba and P. tremula maintain high nuclear and/or plastid divergence in the Irtysh River hybrid zone? 2) What is the genomic composition of hybrids in this zone? 3) Does significant linkage disequilibrium (LD) occur between plastid trnL-trnF variation and the nuclear microsatellite genotype? 4) What evolutionary processes may lead to the CND?

\section{Results}

Polymorphism at microsatellite loci. In each taxon, the number of alleles $(A)$, variance in allele size (Var), expected heterozygosity $\left(H_{\mathrm{E}}\right)$, and observed heterozygosity $\left(H_{\mathrm{O}}\right)$ varied among 11 nuclear microsatellite loci (see Supplementary Table S1). Across all of these loci, both $H_{\mathrm{E}}$ and $H_{\mathrm{O}}$ for $P$. $\times$ canescens $(0.678$ and 0.649 , respectively) were higher than those for P. tremula (0.586 and 0.524$)$ and $P$. alba $(0.360$ and 0.430$)$. Estimates of diversity [allelic richness $\left(A_{\mathrm{r}}\right), H_{\mathrm{E}}$, and $H_{\mathrm{O}}$ ] did not vary greatly across populations within each species, but $P$. tremula populations generally had higher diversity than $P$. alba populations (Table 1$)$. In one $P$. alba population, $F_{\mathrm{IS}}$ was significantly greater than zero, and in two $P . \times$ canescens populations $F_{\text {IS }}$ was significant lower than zero, displaying significant deviations from Hardy-Weinberg equilibrium (HWE) (Table 1). After sequential Bonferroni corrections were applied, significant LD was detected at 28 of 55 pairwise microsatellite locus comparisons in P. tremula, compared with only one pairwise locus comparison in P. alba. Significant LD in $P$. tremula mainly occurred in one population (Pt3). After excluding this population, significant LD was detected at only one pairwise microsatellite locus comparison in P. tremula. Significant LD was detected at 52 of 55 pairwise microsatellite locus comparisons in $P . \times$ canescens.

Differentiation and structure. The differentiation between the two parental species was high across all microsatellite loci, as estimated by both $F_{\mathrm{ST}}(0.418, p<0.001)$ and $R_{\mathrm{ST}}(0.564)$. Many loci exhibited very high differentiation, with seven of the 11 loci displaying both $F_{\mathrm{ST}}$ and $R_{\mathrm{ST}}$ values $>0.3$, only one having an $F_{\mathrm{ST}}<0.1$, and two having an $R_{\mathrm{ST}}<0.1$ (Table 2). The genetic differentiation between hybrid and parental species was lower than that between the parental species, but still moderately high and significant for most loci. However, the hybrid species showed a slightly higher genetic differentiation from $P$. tremula $\left(F_{\mathrm{ST}}=0.182, p<0.001 ; R_{\mathrm{ST}}=0.369\right)$ than 


\begin{tabular}{|c|c|c|c|c|c|c|c|c|c|}
\hline & \multicolumn{7}{|c|}{ Microsatellite } & \multicolumn{2}{|l|}{ trnL-trnF } \\
\hline & $N$ & $A$ & $A_{\mathrm{R}}$ & Var & $H_{\mathrm{o}}$ & $H_{\mathrm{E}}$ & $f$ & $N_{\mathrm{H}}$ (sample size) & $G d$ \\
\hline Pt1 & 23 & 5 & 4.62 & 22.4 & 0.479 & 0.540 & 0.115 & $1(23)$ & 0.000 \\
\hline Pt 2 & 19 & 4 & 4.28 & 22.4 & 0.525 & 0.560 & 0.065 & $2(20)$ & 0.268 \\
\hline Pt 3 & 21 & 4 & 4.23 & 48.1 & 0.573 & 0.531 & -0.081 & $1(21)$ & 0.000 \\
\hline $\mathrm{Pa} 1$ & 20 & 4 & 3.45 & 33.5 & 0.310 & 0.396 & $0.223^{*}$ & $2(20)$ & 0.521 \\
\hline $\mathrm{Pa} 2$ & 20 & 3 & 3.08 & 32.7 & 0.373 & 0.402 & 0.076 & $3(20)$ & 0.416 \\
\hline $\mathrm{Pa} 3$ & 25 & 4 & 3.33 & 30.6 & 0.357 & 0.391 & 0.088 & $3(24)$ & 0.518 \\
\hline $\mathrm{Pa} 4$ & 25 & 5 & 3.92 & 38.9 & 0.396 & 0.458 & 0.139 & $3(23)$ & 0.482 \\
\hline $\mathrm{PaM}$ & 49 & 3 & 2.63 & 27.0 & 0.369 & 0.366 & -0.005 & $4(49)$ & 0.662 \\
\hline Pcl & 6 & 2 & NA & 11.4 & 0.727 & 0.420 & $-0.868^{*}$ & $1(6)$ & 0.000 \\
\hline Pc2 & 25 & 5 & 4.46 & 54.4 & 0.668 & 0.636 & -0.052 & $3(24)$ & 0.301 \\
\hline Pc 3 & 44 & 4 & 3.21 & 43.3 & 0.787 & 0.555 & $-0.427^{*}$ & $3(43)$ & 0.494 \\
\hline Pc 4 & 21 & 5 & 4.89 & 53.8 & 0.575 & 0.583 & 0.013 & $4(18)$ & 0.680 \\
\hline Pc 5 & 18 & 5 & 4.51 & 63.9 & 0.658 & 0.670 & 0.018 & $4(17)$ & 0.419 \\
\hline Pc 6 & 22 & 4 & 3.43 & 39.4 & 0.557 & 0.579 & 0.040 & $2(19)$ & 0.410 \\
\hline
\end{tabular}

Table 1. Genetic variability in each sampled Populus population. $A$, number of alleles; $A_{\mathrm{R}}$, allelic richness; Var, variance in allele size; $H_{\mathrm{O}}$, observed heterozygosity; $H_{\mathrm{E}}$, expected heterozygosity; $f$, inbreeding coefficient; $N_{\mathrm{H}}$, number of plastid DNA haplotypes found in each population; $G d$, gene diversity analogous to $H_{\mathrm{E}}$; ${ }^{*} \mathrm{P}<0.05$.

\begin{tabular}{|l|c|c|c|c|c|r|}
\hline \multirow{2}{*}{ Locus } & \multicolumn{2}{|c|}{ trem vs alba } & \multicolumn{2}{c|}{ trem vs cans } & \multicolumn{2}{c|}{ alba vs cans } \\
\cline { 2 - 7 } & $\boldsymbol{F}_{\boldsymbol{S T}}$ & $\boldsymbol{R}_{\boldsymbol{S T}}$ & $\boldsymbol{F}_{\boldsymbol{S T}}$ & $\boldsymbol{R}_{\boldsymbol{S T}}$ & $\boldsymbol{F}_{\boldsymbol{S T}}$ & \multicolumn{1}{c|}{$\boldsymbol{R}_{\boldsymbol{S T}}$} \\
\hline GCPM1063 & $0.217^{*}$ & 0.489 & $0.073^{*}$ & 0.147 & $0.048^{*}$ & 0.191 \\
\hline GCPM1065 & $0.158^{*}$ & 0.199 & $0.083^{*}$ & 0.057 & $0.020^{*}$ & 0.047 \\
\hline GCPM114 & $0.601^{*}$ & 0.671 & $0.213^{*}$ & 0.206 & $0.190^{*}$ & 0.241 \\
\hline GCPM1158 & $0.720^{*}$ & 0.633 & $0.284^{*}$ & 0.276 & $0.241^{*}$ & 0.173 \\
\hline GCPM124 & $0.444^{*}$ & 0.593 & $0.183^{*}$ & 0.142 & $0.125^{*}$ & 0.199 \\
\hline GCPM1252 & $0.135^{*}$ & 0.081 & $0.051^{*}$ & 0.108 & $0.173^{*}$ & -0.002 \\
\hline GCPM1255 & $0.857^{*}$ & 0.804 & $0.375^{*}$ & 0.330 & $0.324^{*}$ & 0.306 \\
\hline GCPM1260 & $0.317^{*}$ & 0.462 & $0.142^{*}$ & 0.227 & $0.069^{*}$ & 0.227 \\
\hline GCPM1274 & $0.368^{*}$ & 0.775 & $0.343^{*}$ & 0.741 & 0.000 & -0.008 \\
\hline GCPM1353 & $0.095^{*}$ & 0.116 & $0.071^{*}$ & 0.028 & $0.004^{*}$ & 0.027 \\
\hline GCPM139 & $0.360^{*}$ & 0.009 & 0.177 & 0.143 & $0.129^{*}$ & 0.045 \\
\hline All & $0.418^{*}$ & 0.564 & $0.182^{*}$ & 0.369 & $0.120^{*}$ & 0.119 \\
\hline
\end{tabular}

Table 2. Pairwise genetic differentiation between two parental species and their hybrids. trem, Populus tremula; alba, P. alba; cans, $P . \times$ canescens. ${ }^{*} \mathrm{P}<0.05$.

from $P$. alba $\left(F_{\mathrm{ST}}=0.120, p<0.001 ; R_{\mathrm{ST}}=0.119\right)$, consistent with a stronger affinity of hybrids for $P$. alba than for $P$. tremula in the neighbor-joining tree based on the population allelic frequencies of the 11 microsatellite loci (see Supplementary Fig. S1). Within both parental species, nuclear differentiation among populations was low, but relatively higher values were found in $P$. tremula $\left(F_{\mathrm{ST}}=0.105\right.$ and $\left.R_{\mathrm{ST}}=0.151\right)$ than that in $P$. alba $\left(F_{\mathrm{ST}}=0.049\right.$ and $R_{\mathrm{ST}}=0.089$ ).

Analysis of molecular variance (AMOVA) showed that $42.3 \%$ of the nuclear microsatellite variation occurred between $P$. alba and $P$. tremula, resulting in a high statistic for interspecific genetic divergence $\left(F_{\mathrm{CT}}=0.423\right)$. The proportion of variation among populations within each species was small $\left(4.81 \%, F_{\mathrm{SC}}=0.081\right)$, while the proportion of variation within populations followed a complementary pattern $\left(52.86 \%, F_{\mathrm{ST}}=0.571\right.$; Table 3$)$.

Principal coordinate analysis (PCoA) of the pairwise individual genetic distances is presented in Fig. 1 . The first two PCo-axes of the plot accounted for $>65 \%$ of the variation. The plot indicated that P. alba, P. tremula, and $P . \times$ canescens were mostly grouped separately in line with morphological classification in the field, except for several mismatches between hybrids and each parental species; the hybrid species were located between the two parental species, but had a stronger affinity to $P$. alba than to P. tremula. From the plot, compared with the two parental species, $P \times$ canescens showed the largest genotypic variation among individuals, while variation among $P$. alba individuals was greater than that among $P$. tremula individuals, mostly coming from $P$. alba individuals in mixed locations.

Nuclear admixture analysis. STRUCTURE analysis classified all individuals from the Irtysh River hybrid zones into two clusters ( $K=2$, Supplementary Fig. S2). The majority of $P$. alba and $P$. tremula individuals from pure populations were classified into their respective clusters with high admixture coefficients $(Q>0.95)$, and 


\begin{tabular}{|l|c|c|c|c|c|c|c|c|c|c|}
\hline & \multicolumn{9}{|c|}{ Microsatellites } & \multicolumn{5}{c|}{ Plastid DNA } \\
\cline { 2 - 25 } & d.f. & $\begin{array}{c}\text { Sum of } \\
\text { squares }\end{array}$ & $\begin{array}{c}\text { Variation } \\
(\%)\end{array}$ & F-statistic & P & d.f. & $\begin{array}{c}\text { Sum of } \\
\text { squares }\end{array}$ & $\begin{array}{c}\text { Variation } \\
(\%)\end{array}$ & F-statistic & P \\
\hline Between species & 1 & 242.17 & 42.34 & $F_{C T}=0.423$ & $<0.001$ & 1 & 485.168 & 55.75 & $F_{\mathrm{CT}}=0.558$ & $<0.001$ \\
\hline $\begin{array}{l}\text { Among populations } \\
\text { within species }\end{array}$ & 5 & 48.631 & 4.81 & $F_{\mathrm{CS}}=0.083$ & $<0.001$ & 5 & 123.549 & 8.5 & $F_{\mathrm{CS}}=0.192$ & $<0.001$ \\
\hline Within populations & 299 & 585.385 & 52.86 & $F_{\mathrm{ST}}=0.471$ & 0.025 & 142 & 579.122 & 35.75 & $F_{\mathrm{ST}}=0.643$ & 0.026 \\
\hline
\end{tabular}

Table 3. Analysis of molecular variance (AMOVA) for nuclear microsatellite and plastid DNA data at three hierarchical levels. Variance analysis only included the four pure Populus alba and three pure P. tremula populations.

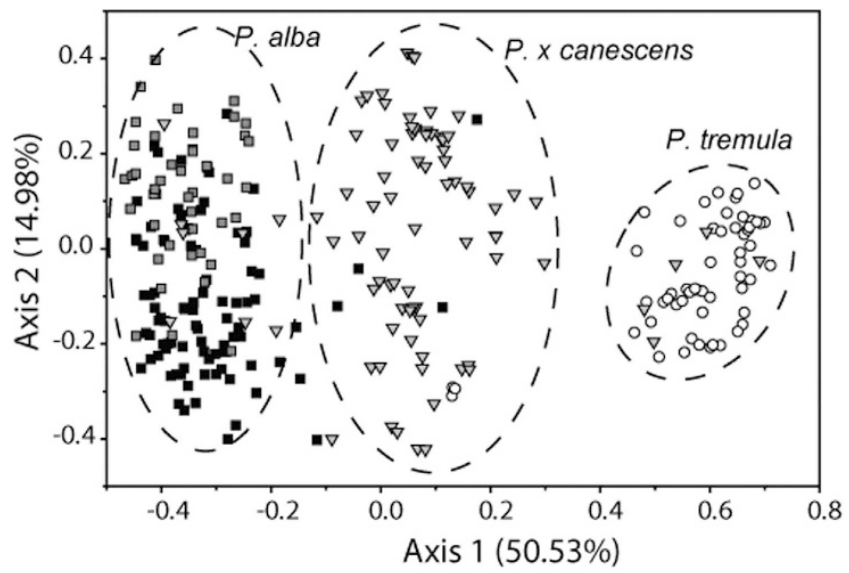

Figure 1. Principal coordinate plots of pairwise differentiation based on 11 nuclear microsatellites for 340 individuals of $P$. alba, $P$. tremula, and $P$. $\times$ canescens. Percentages of total variance explained by each axis are noted in brackets; black squares, $P$. alba from pure sites; grey squares, $P$. alba samples that coexisted with $P . \times$ canescens; triangles, $P . \times$ canescens; circles, $P$. tremula.

(a)

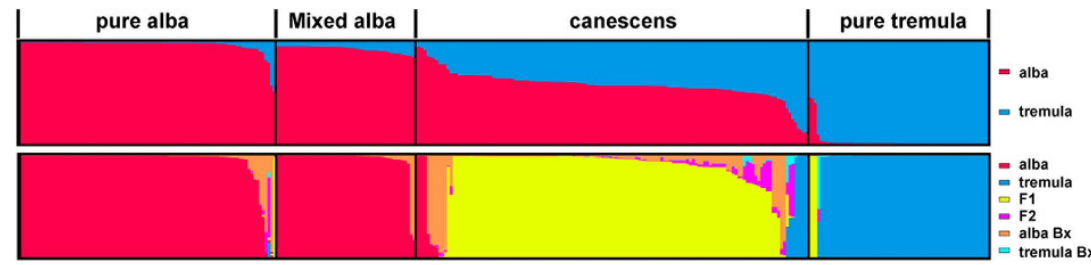

Figure 2. Bayesian clustering results of all sampled Populus individuals based on 11 nuclear microsatellite loci. (a) Individual assignment results using STRUCTURE software at $K=2$. (b) Individual assignment results using NewHybrids software. In all diagrams, each column represents a single individual. The length of each colored segment is proportional to the posterior probability of assignment to P. alba or P. tremula in (a); or is proportional to the posterior probability of assignment to the corresponding genotypic class in (b).

only six P. alba and four P. tremula were classified as hybrids based on their $Q$ values (Fig. 2a). The admixture proportions of $P$. alba individuals from $P$. $\times$ canescens mixed populations ranged from 0.849 to 0.957 , with 23 of 49 individuals $(46.9 \%)$ giving values $>0.95$. In contrast, a wide range of admixture proportions was found for $P$. $\times$ canescens hybrid morphotypes ( $Q$ ranged from 0.107 to 0.055 , with a $95 \%$ confidence interval of $Q=0.348-$ 0.825), indicating the presence of a wide range of hybrid generations and back-crossing to both parental species.

NewHybrids analysis also assigned most $P$. alba and $P$. tremula individuals to their respective clusters with high probabilities ( $>0.75$; Fig. $2 \mathrm{~b})$. In addition, four $P . \times$ canescens individuals were identified as $P$. alba and five as $P$. tremula, with high probability $(>0.98)$. Whereas individuals identified as hybrids in STRUCTURE analysis, including several $P$. alba and $P$. tremula and the majority of $P . \times$ canescens, were mostly assigned to hybrids. Using 0.75 as a threshold, 107 of the $136 P . \times$ canescens individuals and one individual from a $P$. tremula population were identified as F1, eleven $P$. $\times$ canescens individuals and two $P$. alba individuals were identified as back-crossing to $P$. alba, and none were identified as F2 and back-crossing to $P$. tremula. Only two individuals were assigned to F2 and one was assigned to back-crossing to $P$. tremula with probabilities $\sim 0.5$. 


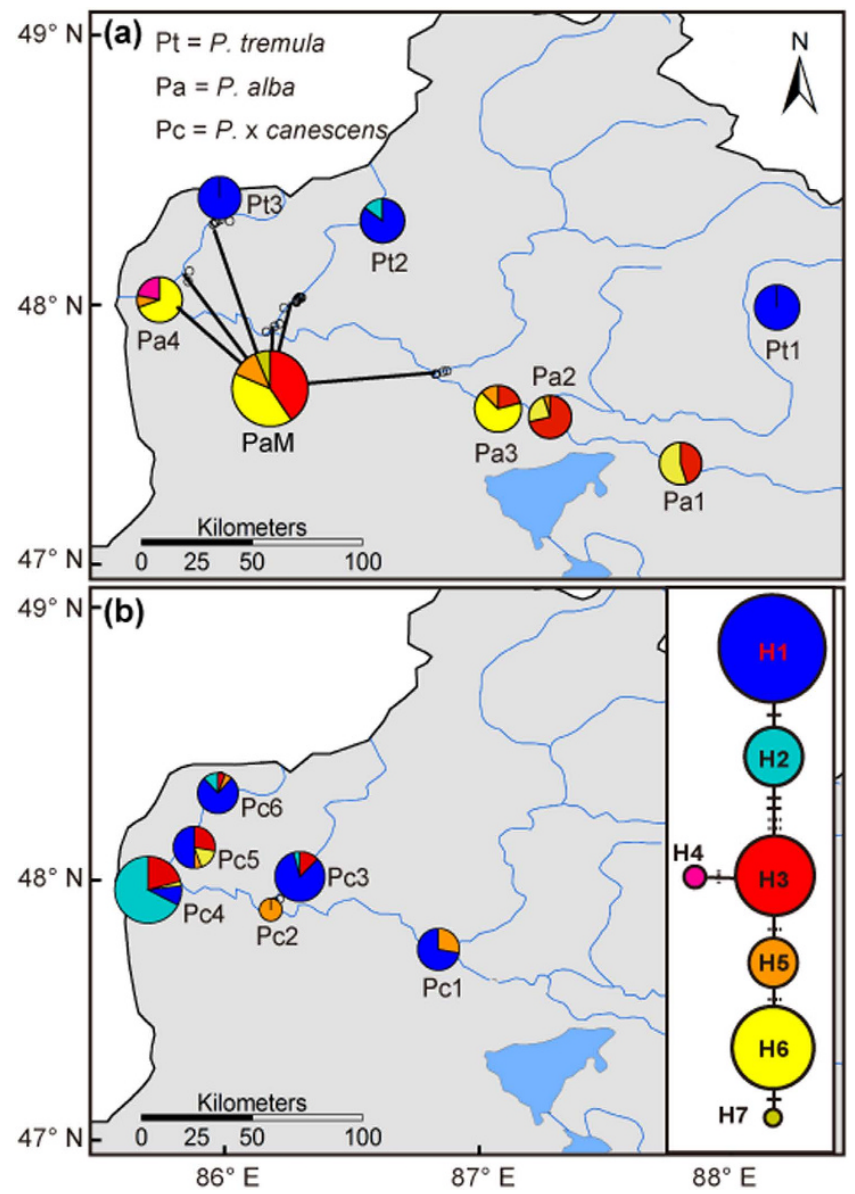

Figure 3. The trnL-trnF haplotype distribution for each Populus population in the Irtysh River hybrid zone. Distributions are shown separately in pure parental species (a) and in hybrid species (b). Circle sizes are proportional to the sample sizes of the populations. Right panel in (b) shows the network of haplotypes; perpendicular tick marks on the lines joining haplotypes represent the number of mutations: dotted ticks, indels; solid ticks, substitutions. The map was created using the ArcMap package in ArcGIS ver. 9.2 (http://www. esri.com/software/arcgis).

Plastid variation and cytonuclear association. Single A/T indel variations in three poly-A/T regions $(443,790$, and $930 \mathrm{bp})$ of $\operatorname{trn} \mathrm{L}-\mathrm{trnF}$ were excluded from analysis because of a potentially high rate of reverse mutation. A matrix of 1004-bp sequences from 325 individuals yielded four nucleotide substitutions and four indel variations, combining into seven haplotypes (H1-H7). Haplotype descriptions are listed in Supplementary Table S2. The sequences of these haplotypes have been deposited in GenBank (accession numbers KT851748KT851754). Two of the haplotypes ( $\mathrm{H} 1$ and $\mathrm{H} 2)$ were detected in $P$. tremula, and the other five (H3-H7) in P. alba (Fig. 3a). Five of the haplotypes (two P. tremula, H1-H2, and three P. alba, H3, H5-H6) were detected in hybrid $P . \times$ canescens (Fig. 3 b). Median-joining analysis resulted in a haplotype network with two major clades connected by three variations, one of which contained $\mathrm{H} 1$ and $\mathrm{H} 2$, while the other consisted of $\mathrm{H} 3-\mathrm{H} 7$ (Fig. 3b).

The gene diversity based on $\operatorname{trn} \mathrm{L}-\mathrm{trn} \mathrm{F}$ for each Populus population varied from 0 (Pt1, Pt3, and Pc1) to 0.680 (Pc 4; Table 1). The total diversity in $P$. alba $\left(h_{\mathrm{T}}=0.642\right)$ was much higher than that in P. tremula $\left(h_{\mathrm{T}}=0.094\right)$, but slightly lower than in the hybrids $\left(h_{\mathrm{T}}=0.692\right)$. AMOVA analysis showed that more than half of the $\operatorname{trn} \mathrm{L}-\operatorname{trn} \mathrm{F}$ sequence variations (55.75\%) occurred between $P$. alba and $P$. tremula, resulting in an $F_{\mathrm{CT}}$ reaching 0.558 ; only $8.50 \%$ of the variation occurred among populations within species $\left(F_{\mathrm{CS}}=0.192\right)$, and $35.5 \%$ of the variation occurred within populations $\left(F_{\mathrm{ST}}=0.643\right.$; Table 3$)$.

The calculation of CND for the whole data set revealed clear deviation from a random distribution between plastid haplotypes and nuclear alleles (D, Table 4): i.e. pure nuclear $P$. tremula genotypes (TT) were significantly associated with the haplotypes of $P$. tremula $(\mathrm{Ht})$, showing significant disequilibria involving homozygous genotypes (D1 and D3); the heterozygous genotypes were also positively associated with the haplotypes of $P$. tremula (D2) at seven of the eight examined nuclear loci. In the group of hybrids, significant positive CND was also found between plastid haplotype and nuclear alleles in three microsatellite loci (D), resulting in significant negative values of D3; a positive value of D1 was found to be significant at one locus and the values were very high at the other four loci $(\mathrm{D} 1=1$, Table 4), although they were not significant due to limited combination counts for statistics. 


\begin{tabular}{|l|c|c|c|c|c|c|c|c|}
\hline \multirow{2}{*}{ Locus } & \multicolumn{4}{|c|}{ Whole data set } & \multicolumn{4}{c|}{ P. $\times$ canescens } \\
\cline { 2 - 8 } & D1 (TT/Ht) & D2 (TA/Ht) & D3 (AA/Ht) & D (T/Ht) & D1 (TT/Ht) & D2 (TA/Ht) & D3 (AA/Ht) & D (T/Ht) \\
\hline GCPM114 & $1.000^{* * *}$ & $0.471^{* * *}$ & $-0.844^{* * *}$ & $0.893^{* * *}$ & 1.000 & $0.331^{* *}$ & $-0.517^{* * *}$ & $0.564^{*}$ \\
\hline GCPM1158 & $0.971^{* * *}$ & $0.393^{* * *}$ & $-0.837^{* * *}$ & $0.882^{* * *}$ & 1.000 & -0.147 & -0.074 & 0.206 \\
\hline GCPM124 & $0.968^{* * *}$ & $0.433^{* * *}$ & $-0.774^{* * *}$ & $0.833^{* * *}$ & 1.000 & 0.001 & -0.122 & 0.217 \\
\hline GCPM1252 & $0.835^{* * *}$ & $0.636^{* * *}$ & $-0.745^{* * *}$ & $0.777^{* * *}$ & $0.480^{* *}$ & -0.342 & $-0.582^{*}$ & $0.516^{* * *}$ \\
\hline GCPM1255 & $0.970^{* * *}$ & $0.307^{* * *}$ & $-0.884^{* * *}$ & $0.914^{* * *}$ & 0.355 & 0.220 & -0.457 & 0.434 \\
\hline GCPM1260 & $0.506^{* * *}$ & $0.250^{* * *}$ & $-0.818^{* * *}$ & $0.654^{* * *}$ & 0.052 & 0.040 & -0.209 & 0.118 \\
\hline GCPM1274 & $0.839^{* * *}$ & -0.043 & $-0.632^{* * *}$ & $0.721^{* * *}$ & 0.417 & 0.599 & -0.494 & 0.466 \\
\hline GCPM139 & $1.000^{* * *}$ & $0.659^{* * *}$ & $-0.823^{* * *}$ & $0.874^{* * *}$ & 1.000 & $0.554^{* * *}$ & $-0.617^{* * *}$ & $0.635^{* * *}$ \\
\hline
\end{tabular}

Table 4. Cytonuclear disequilibria in Irtysh River hybrid zones of Populus tremula and P. alba. D1, D2, D3, genotypic disequilibria; D, allelic disequilibria; TT, nuclear genotypes typical of $P$. tremula; TA, nuclear genotypes heterozygous for T and A alleles; AA, nuclear genotypes typical of $P$. alba; Ht, plastid DNA haplotype typical of $P$. tremula; Fisher's exact test: ${ }^{*} 0.01<\mathrm{P}<0.05,{ }^{* *} 0.001<\mathrm{P}<0.01,{ }^{* * *} \mathrm{P}<0.001$.
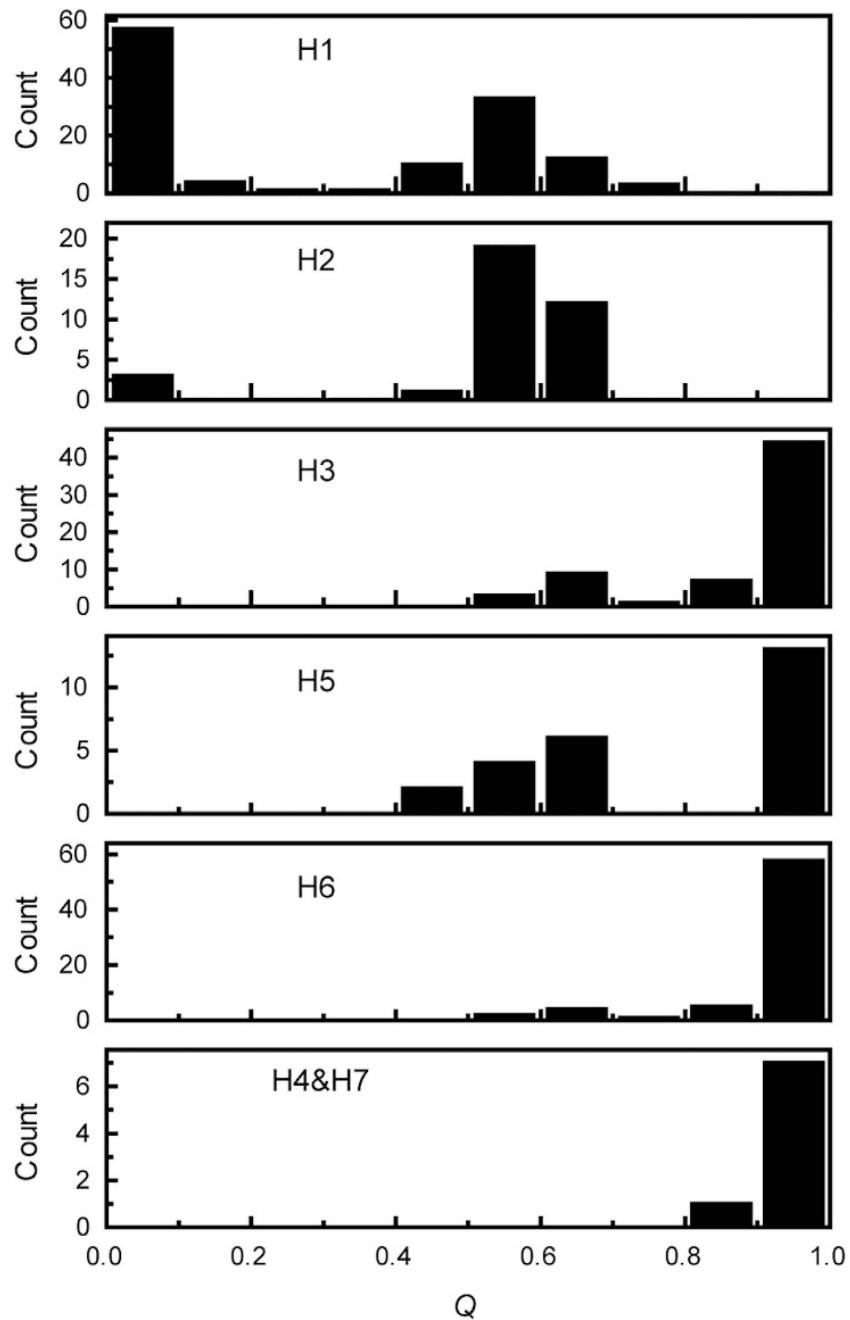

Figure 4. Distribution of estimates of admixture proportion in each $\operatorname{trnL}$-trnF haplotype. The admixture proportion is based on the posterior probability of belonging to $P$. alba $(Q)$ in STRUCTURE analysis (for $K=2$ ) using the 11 nuclear microsatellites.

A simple test for correlation between $\operatorname{trn} \mathrm{L}-\operatorname{trn} \mathrm{F}$ haplotypes and nuclear genomic composition ( $Q$ estimated by STRUCTURE analysis) yielded significant results both for all individuals (Spearman's $\mathrm{r}=0.794, \mathrm{P}<0.001$ ) and after excluding $P$. alba and $P$. tremula populations (Spearman's $\mathrm{r}=0.675, \mathrm{P}<0.001$ ). The distribution of admixture proportion $(Q)$ in each haplotype showed that the range of $Q$ varied greatly among haplotypes (Fig. 4). Haplotype 
$\mathrm{H} 1$ mainly occurred in pure $P$. tremula and hybrids, with a wide range of $Q$ from 0 to $0.8 ; \mathrm{H} 2$ mainly occurred in hybrids with $Q$ ranging between 0.4 and 0.7 , and was also found in several $P$. tremula individuals; $\mathrm{H} 3$ and H6 mainly occurred in pure $P$. alba and were found in a few hybrids with $Q>0.5$. H5 occurred in pure $P$. alba and hybrids with a $Q$ value between 0.4 and 0.7 , while $\mathrm{H} 4$ and $\mathrm{H} 7$ only occurred in individuals showing $P$. alba ancestry.

\section{Discussion}

P. alba and P. tremula are model species in which to study the later stages of tree speciation, and the maintenance of species identity with gene flow ${ }^{36}$. Our current analysis of the $P$. alba and $P$. tremula hybrid zone along the Irtysh River further supports the concept that high hybridization rates and appreciable hybrid fitness do not necessarily conflict with the maintenance of species integrity ${ }^{31}$. First of all, the results from both PCoA and STRUCTURE analysis showed that, in the Irtysh River region, $P$. alba and $P$. tremula both maintained their own species identity, although the hybrid $P . \times$ canescens was prevalent. The parental species and their hybrids were separated into three distinguishable groups in the PCoA plot, consistent with previous analysis of these three species in the European hybrid zones ${ }^{27,28}$. Meanwhile, consistent with the high interspecific $F_{\mathrm{ST}}$ values found in the European contact zone using similar markers $\left(F_{\mathrm{ST}}=0.37\right)^{37}$, the mean genetic differentiation across the 11 microsatellites was high between the two parental species in the Irtysh River hybrid zone $\left(F_{\mathrm{ST}}=0.418\right.$ and $\left.R_{\mathrm{ST}}=0.564\right)$, reflecting their long divergence time and limited interspecific gene flow ${ }^{28}$. Furthermore, most of these microsatellite loci showed high interspecific differentiation, and the few loci that showed a low genetic differentiation may be a result of shared ancestry polymorphism or gene-flow between species ${ }^{37}$. Notably, STRUCTURE analysis that is based on the hypothesis of HWE found only two genetic clusters rather than three (Supplementary Fig. S2), suggesting that the hybrid $P . \times$ canescens has not yet formed a completely distinct gene pool. Notably, we found that population differentiation within parental species was relatively higher in $P$. tremula $\left(F_{\mathrm{ST}}=0.105\right.$ and $\left.R_{\mathrm{ST}}=0.151\right)$ than that in $P$. alba $\left(F_{\mathrm{ST}}=0.049\right.$ and $\left.R_{\mathrm{ST}}=0.089\right)$, which is contrary to the results from the European populations of these species, where much lower differentiation was always found among populations of $P$. tremula ${ }^{28}$. The higher differentiation of $P$. tremula in our current analysis was mainly caused by population Pt3, which was located at a lower altitude than other two $P$. tremula populations (see Supplementary Table S3). In this population, significant LD was detected at 20 of 55 pairwise microsatellite locus comparisons, indicating assortative mating or selection on certain genotype combinations. Further analysis of this population is needed to understand the adaptive evolution of $P$. tremula.

Consistent with the results of a recent study from the European hybrid zones ${ }^{32}$, our current analysis also found that F1 hybrids prevailed in the Irtysh River hybrid zone; however, due to differences in analytical methods, the hybrid $P$. $\times$ canescens had previously been considered to be highly recombinant ${ }^{25,27,28}$. Their prevalence in both of these geographically distant hybrid zones are unlikely to be strongly affected by stochastic or locality-specific events, but rather reflect the high fitness of such genomic patterns of hybrids due to heterosis ${ }^{28,31,32}$. Although the wide range of $Q$ values in STRUCTURE analysis based on nuclear microsatellites indicated that hybrids could back-cross towards both parental species in the Irtysh River hybrid zone (Fig. 2a), NewHybrids analysis found almost no F2 hybrids and back-cross hybrids towards P. tremula (Fig. 2b). It is possible that the NewHybrids analysis could not discriminate between pure $P$. tremula and back-crosses due to limited microsatellite loci ${ }^{38}$, so some hybrids with an ancestry close to $P$. tremula were assigned to the pure species (Fig. 2b). Meanwhile, a low frequency of back-cross hybrids was evident from the results of both STRUCTURE and NewHybrids analysis (Fig. 2). We suggest that strong viability selection from seedling to adult may be responsible for the sparseness of later-generated hybrids and this may contribute to the maintenance of high species differentiation with hybridization in Populus ${ }^{31,32}$. Especially, weak reproductive isolation between the two species and their hybrids was suggested by the presence of the full range of hybrids in seedlings in the European hybrid zones ${ }^{31}$, and have been found in our hand-pollinated hybridization as well (unpublished data). However, in contrast to those hybrids back-crossing toward P. tremula, more back-cross hybrids toward P. alba were detected in NewHybrids analysis. This is reasonable, as $P . \times$ canescens coexisted with $P$. alba, and so had a higher possibility of back-crossing with it. The preponderance of F1 hybrids was also supported by the PCoA plot (Fig. 1), in which most hybrids were intermediate between the two parental species. While estimation of genetic differentiation, the PCo plot, and the population neighbor-joining tree all suggested that hybrids had a slightly stronger affinity to $P$. alba than to $P$. tremula, reflecting preferential introgression back-crosses towards $P$. alb $a^{24,27,28}$. It is also possible that our inferred F1 hybrids carried older traces of recombination not visible with limited genetic markers in current study; whereas older admixture pulses were detected in European hybrid zone using high-density single-nucleotide polymorphisms (SNPs) ${ }^{32}$.

A previous study on hybrid zones of $P$. tremula and $P$. alba in the Danube valley found that the hybrid $P$. $\times$ canescens preferentially carried the plastid DNA of $P$. alba, indicating that hybridization occurred preferentially via $P$. tremula pollen and $P$. alba seed parents ${ }^{24}$. Lexer et al. ${ }^{24}$ suggested that this unidirectional pattern is facilitated by high levels of pollen versus seed dispersal in $P$. tremula and by great ecological opportunity in the lowland flood-plain forest near $P$. alba seed parents. However, our current analysis showed that $P . \times$ canescens carried the plastid DNA of both $P$. tremula and $P$. alba, and even more hybrids carried the plastid DNA of the former, reflecting positive associations between the heterozygous genotypes and haplotypes of $P$. tremula (D2, Table 4). This means that, in the Irtysh River valley, hybridization could occur via $P$. alba pollen and $P$. tremula seed parents, as well as via P. tremula pollen and P. alba seed parents. During the sampling, we found that in this zone, hybrids mostly occurred in the flood-plains of tributaries or the flood-plains where tributaries join the main river; only a few occurred in the flood-plain of the main river, where $P$. alba was prevalent. Thus, it is possible that the hybrid seeds from $P$. tremula that grow in the upland are dispersed to the lowland flood-plain by wind or by the flow of melt-water along the tributaries of the Irtysh River every spring ${ }^{35}$. 
Unlike potential nuclear introgression, the plastid $\operatorname{trn} \mathrm{L}-\mathrm{trnF}$ sequence was completely distinct between the two parental species in the Irtysh River hybrid zone (Fig. 3a). Significant CNDs across all loci were found in the whole dataset, demonstrating a non-random association of the nuclear genome with the plastid genomes in this Populus hybrid zone (Table 4). Departures from random associations between cytoplasmic and nuclear genetic markers in hybrids can reflect the migration of parental genotypes into the hybrid populations, assortative mating among similar genotypes, selection due to cytonuclear interactions, or cytoplasmic male sterility (see references in Cruzan \& Arnold ${ }^{23}$ ). Factors like assortative mating and migration can generate a CND de novo, and they are likely to affect all chromosomes ${ }^{39}$. Strong CND observed consistently across loci for the whole data set might be driven by migration and assortative mating of parental genotypes in the large hybrid zone. Especially, P. alba and $P$. tremula are distributed separately in river basins and in mountainous regions, respectively, thus mating tends to occur within each species.

However, within the hybrid $P . \times$ canescens group, the plastid of $P$. tremula was also significant associated with nuclear alleles of $P$. tremula in several but not all microsatellite loci (Table 4). Significant CND between the plastid and a few nuclear loci in $P . \times$ canescens has also recently been found in the European hybrid zone ${ }^{33}$. The contribution of parental conspecifics to CND by migration could be neglected, as only hybrids were included in the analysis; assortative mating, however, is not possible for wind-pollinated Populus when the flowering times of the two parental species largely overlap ${ }^{33}$ and hybrids with different haplotypes always coexisted in the same forest (Fig. 3b). It is possible that such a CND pattern in hybrids is caused by the action of selection on interspecific combinations of cytoplasmic and nuclear genotypes due to incompatibility. Unlike CND caused by assortative mating and migration that affect all chromosomes, that caused by selection is more locus-specific ${ }^{22}$; only the microsatellite markers linked to certain genes involved in cytonuclear processes might cause the observed disequilibria, especially in early-generation hybrids carrying relatively large chromosome blocks inherited from each parental species ${ }^{33}$.

Selection against certain hybrid classes has been identified in the European Populus hybrid zone ${ }^{31,32}$, and was also supported by the low frequency of post-F1 hybrids in the current zone. More important, the significant association between plastid haplotypes and the proportion of nuclear admixture in hybrids (Spearman's $\mathrm{r}=0.675, \mathrm{P}<0.001)$ suggested that the haplotype distribution in different hybrid classes was also non-random: the $P$. tremula haplotypes tended to occur in hybrids with a reduced genomic composition of $P$. alba $(Q<0.8)$; $P$. alba haplotypes only occurred in hybrids with a reduced genomic composition of $P$. tremula $(Q>0.4)$, demonstrating a pattern of interspecific plastid-nuclear incompatibility (Fig. 4). Therefore, we infer that the "breakdown" of fitness by incompatibility might have resulted in selection against later-generated hybrids carrying interspecific combinations of cytoplasmic and nuclear genotypes and finally exhibited significant linkage between the plastid genome and certain nuclear loci.

What to mention, a recent study from European hybrid zones inferred that cytonuclear interactions were less likely to contribute to genomic isolation of these poplar in three studied localities, where cytoplasms of each species appeared to combine freely with different nuclear genomes ${ }^{32}$. Consistent with results of their earlier study ${ }^{24}$, Christe et al. ${ }^{32}$ found that hybrids in these localities tended to carry the plastid genome of their spatially closer parent. It is reasonable, as the plastid genome of poplar is seed-mediated and seeds are less mobile than their pollen. However, the spatial distribution of hybrids could not fully explain the observed plastid-nuclear pattern in the Irtysh River hybrid zone, where $P . \times$ canescens individuals $(\mathrm{Pc})$ coexisted with $P$. alba (PaM in Fig. 3a) and carried haplotypes of either parents, no matter where they located (Fig. 3b). Of cause, to confirm the role of cytonuclear interactions on the observed plastid-nuclear pattern of hybrids, more hybrid samples are needed to exclude the influence of a spatial distribution, especially those locate close to P. tremula, and more markers are necessary to scale the nuclear composition of hybrids more accurately.

In all, our results showed that plastid-nuclear incompatibility might have established hybridization barriers between parental species and hybrids in the Irtysh River hybrid zone and finally influenced the extent and pattern of introgression between P. tremula and P. alba. However, to further assess the role of plastid-nuclear incompatibility in the speciation of Populus, more genetic information on the plastid genomes of $P$. tremula and $P$. alba are needed to allow comparison of the adaptive divergence of related proteins between species. Also, hand-pollination studies are needed to compare the hybrid variegation among different plastid and nuclear genome combinations. In the current analysis, we only chose markers within the plastid genome, and thus only considered the association between plastid and nuclear genomes. Meanwhile, the influence of the mitochondrial genome cannot be excluded, as it is also maternally inherited in Populus and thus linked to the plastid genome ${ }^{34,40}$.

\section{Materials and Methods}

Study sites and sampling. The two parental species, $P$. alba (white poplar) and $P$. tremula (European aspen), and the hybrid $P . \times$ canescens (gray poplar) were sampled from the Irtysh River system from $85^{\circ} \mathrm{E}$ to $88^{\circ} \mathrm{E}$, including the main Irtysh River and three tributaries: the Haba, Buerjin, and Buliezi Rivers. In this region, pure $P$. alba populations were mainly found along the main Irtysh River; pure P. tremula populations were located in mountainous regions near tributaries; and $P . \times$ canescens prevailed along the tributaries with a few coexisting P. alba.

Individuals were classified into different taxa in the field based on leaf morphology, such as shape and abaxially white tomentose, following the Flora of China ${ }^{41}$. In total, 63 P. tremula individuals were sampled from three pure populations (Pt1-3) and $90 \mathrm{P}$. alba individuals were sampled from four pure populations (Pa1-4). In addition, $136 \mathrm{P} . \times$ canescens individuals and $49 \mathrm{P}$. alba individuals $(\mathrm{PaM})$ that coexisted along the three river tributaries were sampled from 28 sites (see Supplementary Table S3). For analysis, hybrid individuals from these sites were clustered into six populations by combining nearby sites (Pc1-6, see Supplementary Fig. S3). The sampled trees were at least $50 \mathrm{~m}$ distant from each other. Leaves were dried using silica gel and then taken back to the laboratory for DNA extraction. 
Microsatellite marker procedure. The samples were screened for variation at 11 nuclear microsatellite loci supplied by the International Populus Genome Consortium (http://www.ornl.gov/sci/ipgc/ssr_resource. $\mathrm{htm}$ ), see Supplementary Table S4 for primer details. The 11 microsatellites were amplified by the polymerase chain reaction (PCR) following the methods described by He et al. ${ }^{42}$, with each forward primer labeled with one of the fluorescent dyes 6-FAM, HEX, or TAMRA (Sangon Biotech, Shanghai, China).

The microsatellite genotypes of all loci were resolved on an ABI 3130xl automated sequencer (Life Technologies, Foster City, CA), making use of the different fluorescent dyes and size differences between loci for multiplexing. Molecular sizes in base pairs were determined using the GENESCAN-500 ROX size standard. The microsatellite genotyping was subsequently analyzed using GENEMAPPER software version 3.7 (Life Technologies) and visually checked twice. Each set of 48 reactions included a positive (known genotype) and a negative (water) control carried from PCR through to the final automated sequencer analysis of microsatellites.

Plastid sequence procedure. The plastid intergenic spacer $\operatorname{trn} \mathrm{L}-\operatorname{trn} \mathrm{F}$ region was amplified using the primers trnLF-f ( $5^{\prime}$-ATTTGAACTGGTGACACGAG- $\left.3^{\prime}\right)$ and $t r n$ LF-r ( $5^{\prime}$-CGAAATCGGTAGACGCTACG-3'), which were improved from the original of Taberlet $e t a l .^{43}$, based on the complete genome of the $P$. trichocarpa chloroplast (GenBank accession number NC_009143 ${ }^{44}$ ). PCR was performed in a $25-\mu \mathrm{L}$ volume, containing $10 \mathrm{mM}$ Tris- $\mathrm{HCl}(\mathrm{pH} 8.0), 0.2 \mu \mathrm{M}$ of each dNTP, $1.5 \mathrm{mM} \mathrm{MgCl}_{2}, 0.16 \mu \mathrm{M}$ of each forward and reverse primer, $1 \mathrm{U}$ Taq DNA polymerase (TaKaRa, Tokyo, Japan), and $1 \mu \mathrm{l}(10-50 \mathrm{ng})$ genomic DNA. PCRs were performed in an Eppendorf Mastercycler, programmed for initial denaturing at $94^{\circ} \mathrm{C}$ for $4 \mathrm{~min}$, followed by 30 cycles of denaturing for $45 \mathrm{~s}$ at $94^{\circ} \mathrm{C}$, annealing at $60^{\circ} \mathrm{C}$ for $45 \mathrm{~s}$, and extension for $1 \mathrm{~min}$ and $20 \mathrm{~s}$ at $72^{\circ} \mathrm{C}$. A final extension followed at $72^{\circ} \mathrm{C}$ for $8 \mathrm{~min}$. PCR products were purified with a Quick PCR Purification Kit (Tiangen, Beijing, China), and then all DNA sequencing was performed using an ABI Prism Bigdye ${ }^{\mathrm{TM}}$ terminator cycle sequencing ready reaction kit (Life Technologies, Foster City, CA). The reaction mixtures were analyzed on an ABI 3130xl automated sequencer (Life Technologies).

Nuclear data analysis. Nuclear genetic diversity and differentiation. In order to characterize the microsatellite loci in the two study species and their hybrids, the number of alleles $(A)$, variance in allele size (Var), expected heterozygosity $\left(H_{\mathrm{E}}\right)$, and observed heterozygosity $\left(H_{\mathrm{O}}\right)$ were calculated for each locus. In each population, $V a r$, allelic richness $\left(A_{\mathrm{r}}\right), H_{\mathrm{E}}$, and $H_{\mathrm{O}}$ were calculated across all microsatellite loci. All analyses were performed using the MSA program ${ }^{45}$. The inbreeding coefficient $F_{\text {IS }}$ was calculated using FSTAT $^{46}$ and the significance of $F_{\text {IS }}$ was tested for departures from HWE for each population after Bonferroni correction. LD among microsatellite loci was analyzed for each species using the log-likelihood ratio statistic (G-test) implemented in Genepop $4.3^{47}$ with the default set of Markov chain parameters and the nominal level of statistical significance set at 0.01 .

The genetic differentiation between species or among populations within species was estimated by $F_{\mathrm{ST}}{ }^{48}$ and $R_{\mathrm{ST}}{ }^{49}$ for the 11 microsatellite loci using the program FSTAT $2.9 .3^{46}$. The significance of $F_{\mathrm{ST}}$ was tested by comparison of the observed $F_{\mathrm{ST}}$ with a distribution of $F_{\mathrm{ST}}$ under the hypothesis of no genetic structure, obtained by means of 5,000 random permutations of individuals between species or among populations.

AMOVA in Arlequin $3.11^{50}$ was used to obtain F-statistics for nuclear microsatellite markers between the two parental species. We tested the hierarchies "among species", "among populations within species", and "within populations" using only pure populations of $P$. alba and $P$. tremula.

PCoA analysis was performed for microsatellite data sets to calculate principal co-ordinates from pairwise Euclidian distance estimates between individual genotypes. Analyses were executed in GenAlEx $6^{51}$. The first two axes were plotted graphically with Origin 7.5 (OriginLab, Northampton, MA).

Nuclear admixture analysis. To identify the possible genetic structure and admixture of individuals in the hybrid zone, we used a model-based Bayesian approach implemented in STRUCTURE ver. $2.3 .3^{52,53}$. The program was run, without prior population information, under the admixture model allowing for correlated allele frequencies. Ten independent replicates for each $K$ were analyzed for $K=1-7$, setting burn-in and run lengths of 500000 and 2000000 iterations, respectively. The final posterior probability of $K, \operatorname{Pr}(X \mid K)$, and delta $K(\Delta K)$, where the modal value of the distribution is located at the real $K^{54}$, were used to determine the most likely number of clusters.

The method developed by Anderson and Thompson ${ }^{55}$ and performed in NewHybrids was used to achieve a more detailed analysis of admixture proportions and hybrid ancestry by inferring the posterior probability assignment $(Q)$ of each sampled individual to one of six genotype frequency classes: P. tremula, P. alba, F1 (first generation hybrids), F2 (second generation hybrids, crossing of F1 $\times$ F1), B $\times$ tremula (back-cross of F1 hybrids towards pure $P$. tremula) and $\mathrm{B} \times$ alba (back-cross of $\mathrm{F} 1$ hybrids towards pure $P$. alba). The program was run with the nuclear microsatellite data using 50000 burn-in iterations followed by 500000 Markov chain Monte Carlo iterations using default priors for allele frequencies and mixing proportions.

Plastid DNA analysis. The sequences of $\operatorname{trn} \mathrm{L}-\operatorname{trn} \mathrm{F}$ were first aligned with Clustal $\mathrm{X}^{56}$ and then manually adjusted. A matrix of combined sequences was constructed for the 325 individuals that we examined and different plastid sequences were identified as haplotypes. A median-joining network ${ }^{57}$ was constructed between haplotypes using the program Network ver. 4.6.1.3. (www.fluxus-engineering.com).

Gene diversity, the probability that two randomly-chosen homologous sites are different, was analyzed for both populations and species using ARLEQUIN ver. $3.1^{58}$. With the same program, AMOVA was used to obtain F-statistics for among species, among populations within species, and within populations, using plastid sequences of only pure $P$. alba and $P$. tremula populations.

Plastid-nuclear LD was estimated between each nuclear microsatellite locus and the plastid trnL-trnF locus by testing departures from random cytonuclear associations ${ }^{22}$ using the CNDm program ${ }^{59}$. The analyses were 
carried out by encoding nuclear markers in the form of synthetic alleles ( $\mathrm{T}$, alleles typical of $P$. tremula; A, alleles typical of $P$. alba) and by encoding plastid DNA alleles as synthetic haplotypes ( $\mathrm{Ht}$, haplotypes typical of $P$. tremula; Ha, haplotypes typical of P. alba). Species-specific nuclear alleles were defined based on both clear frequency differences and diagnostic alleles. The loci GCPM1063, GCPM1065, and GCPM1353, whose common alleles did not show clear frequency differences between the two species, were excluded from analysis.

The normalized CND was calculated for the whole dataset and for the hybrid $P . \times$ canescens only, following Asmussen and Basten ${ }^{60}$ for allelic and genotypic associations, and significance levels were tested using Fisher's exact test. Significantly positive and negative values of CND indicate positive and negative associations between nuclear and cytoplasmic genomes, respectively. Significant disequilibria involving heterozygous nuclear loci (TA) - represented by D2 (TA/Ht) - point to nonrandom mating in hybrid zones and unidirectional hybridization, whereas significant disequilibria involving homozygous genotypes D1 and D3 (involving nuclear genotypes $\mathrm{TT}$ and AA, respectively) point to barriers to introgression, effectively maintaining species integrity in the face of gene flow ${ }^{39}$.

To test for a possible role of plastid-nuclear incompatibilities in determining the patterns of introgression across the hybrid zone, the proportion of nuclear admixture $(Q)$ estimated by the STRUCTURE program at $K=2$ and plastid haplotype data were compared in a simple way: nonparametric Spearman rank correlations were used to test for a possible association between nuclear and plastid genomic composition using SPSS (SPSS Inc., Chicago, IL). The test was done both for all individuals and for the hybrid $P . \times$ canescens only. The distribution of nuclear admixture proportions was demonstrated for each $\operatorname{trn} \mathrm{L}-\operatorname{trn} \mathrm{F}$ haplotype with Origin 7.5 (OriginLab, Northampton, MA).

\section{References}

1. Nosil, P. Ecological speciation. (Oxford University Press, 2012).

2. Abbott, R. et al. Hybridization and speciation. J. Evol. Biol. 26, 229-246 (2013).

3. Mallet, J., Beltrán, M., Neukirchen, W. \& Linares, M. Natural hybridization in heliconiine butterflies: the species boundary as a continuum. BMC Evol. Biol. 7, 28 (2007).

4. Wu, C.-I. The genic view of the process of speciation. J. Evol. Biol. 14, 851-865, doi: 10.1046/j.1420-9101.2001.00335.x (2001).

5. Schluter, D. Ecology and the origin of species. Trends Ecol. Evol. 16, 372-380 (2001).

6. Lynch, M. The genetic interpretation of inbreeding depression and outbreeding depression. Evolution 45, 622-629 (1991).

7. Landry, C. R., Hartl, D. L. \& Ranz, J. M. Genome clashes in hybrids: insights from gene expression. Heredity 99, 483-493 (2007)

8. Edmands, S. \& Timmerman, C. C. Modeling Factors Affecting the Severity of Outbreeding Depression. Conserv. Biol. 17, 883-892 (2003).

9. Orr, H. A. Dobzhansky, Bateson, and the genetics of speciation. Genetics 144, 1331-1335 (1996).

10. Gavrilets, S. Perspective: models of speciation: what have we learned in 40 years? Evolution 57, 2197-2215 (2003).

11. Turelli, M. \& Orr, H. A. Dominance, epistasis and the genetics of postzygotic isolation. Genetics 154, 1663-1679 (2000).

12. Burton, R. S., Pereira, R. J. \& Barreto, F. S. Cytonuclear genomic interactions and hybrid breakdown. Annu. Rev. Ecol. Evol. Syst. 44, 281-302 (2013).

13. Greiner, S., Rauwolf, U. W. E., Meurer, J. \& Herrmann, R. G. The role of plastids in plant speciation. Mol. Ecol. 20, 671-691, doi: 10.1111/j.1365-294X.2010.04984.x (2011).

14. Levin, D. A. The cytoplasmic factor in plant speciation. Syst. Bot. 28, 5-11 (2003).

15. Kirk, J. T. O. \& Tilney-Bassett, R. A. E. The plastids, their chemistry, structure, growth, and inheritance. 2 cd edn, 1-960 (Elsevier, 1978).

16. Stubbe, W. The role of the plastome in evolution of the genus Oenothera. Genetica 35, 28-33 (1964).

17. Fishman, L. \& Willis, J. H. A cytonuclear incompatibility causes anther sterility in Mimulus hybrids. Evolution 60, 1372-1381 (2006).

18. Greiner, S. Oenothera, a unique model to study the role of plastids in speciation Doctor thesis, Ludwig-Maximilians-Universität München (2008).

19. Hewitt, G. M. Hybrid zones-natural laboratories for evolutionary studies. Trends Ecol. Evol. 3, 158-167 (1988).

20. Nagano, Y., Hirao, A. S. \& Itino, T. Genetic structure of a hybrid zone between two violets, Viola rossii Hemsl. and V. bissetii Maxim.: dominance of F1 individuals in a narrow contact range. Plant Species Biol. 30 (2014).

21. Wu, C. A. \& Campbell, D. R. Cytoplasmic and nuclear markers reveal contrasting patterns of spatial genetic structure in a natural Ipomopsis hybrid zone. Mol. Ecol. 14, 781-792 (2005).

22. Arnold, J. Cytonuclear disequilibria in Hybrid Zones. Annu. Rev. Ecol. Syst. 24, 521-554, doi: 10.2307/2097189 (1993).

23. Cruzan, M. B. \& Arnold, M. L. Consequences of cytonuclear epistasis and assortative mating for the genetic structure of hybrid populations. Heredity 82, 36-45 (1999).

24. Lexer, C., Fay, M. F., Joseph, J. A., Nica, M. S. \& Heinze, B. Barrier to gene flow between two ecologically divergent Populus species, P. alba (white poplar) and P. tremula (European aspen): the role of ecology and life history in gene introgression. Mol. Ecol. 14, 1045-1057 (2005).

25. Lexer, C. et al. The use of digital image-based morphometrics to study the phenotypic mosaic in taxa with porous genomes. Taxon 52, 349-364 (2009).

26. Lindtke, D., González-Martínez, S., Macaya-Sanz, D. \& Lexer, C. Admixture mapping of quantitative traits in Populus hybrid zones: power and limitations. Heredity 111, 474-485 (2013).

27. Lexer, C. et al. Genomic admixture analysis in European Populus spp. reveals unexpected patterns of reproductive isolation and mating. Genetics 186, 699 (2010).

28. Lindtke, D. et al. Recombinant hybrids retain heterozygosity at many loci: new insights into the genomics of reproductive isolation in Populus. Mol. Ecol. 21, 5042-5058 (2012).

29. van Loo, M., Joseph, J. A., Heinze, B., Fay, M. F. \& Lexer, C. Clonality and spatial genetic structure in Populus x canescens and its sympatric backcross parent P-alba in a Central European hybrid zone. New Phytol. 177, 506-516 (2008).

30. Lexer, C., Buerkle, C. A., Joseph, J. A., Heinze, B. \& Fay, M. F. Admixture in European Populus hybrid zones makes feasible the mapping of loci that contribute to reproductive isolation and trait differences. Heredity 98, 74-84 (2007).

31. Lindtke, D., Gompert, Z., Lexer, C. \& Buerkle, C. A. Unexpected ancestry of Populus seedlings from a hybrid zone implies a large role for postzygotic selection in the maintenance of species. Mol. Ecol. 23, 4316-4330 (2014).

32. Christe, C. et al. Selection against recombinant hybrids maintains reproductive isolation in hybridizing Populus species despite F1 fertility and recurrent gene flow. Mol. Ecol. In press (2016).

33. Fussi, B. Phylogeography, flowering phenology and cytonuclear interactions of Populus alba and P. tremula Doctor thesis, University of Wien (2010).

34. Rajora, O. \& Dancik, B. Chloroplast DNA inheritance in Populus. Theor. Appl. Genet. 84, 280-285 (1992).

35. Zhang, J. G., Zeng, Y. F. \& He, C. Y. Origin and Evolution of Populus species in Xinjiang. (Chinese Forestry Press, 2014). 
36. Gailing, O. Strategies to Identify Adaptive Genes in Hybridizing Trees like Oaks and Poplars. Not. Bot. Horti. Agrob. 42, 299-309 (2014).

37. Stölting, K. N. et al. Genomic scan for single nucleotide polymorphisms reveals patterns of divergence and gene flow between ecologically divergent species. Mol. Ecol. 22, 842-855 (2013).

38. VÄHÄ, J.-P. \& Primmer, C. R. Efficiency of model-based Bayesian methods for detecting hybrid individuals under different hybridization scenarios and with different numbers of loci. Mol. Ecol. 15, 63-72, doi: 10.1111/j.1365-294X.2005.02773.x (2006).

39. Asmussen, M. A., Arnold, J. \& Avise, J. C. The effects of assortative mating and migration on cytonuclear associations in hybrid zones. Genetics 122, 923-934 (1989)

40. Rajora, O., Barrett, J., Dancik, B. \& Strobeck, C. Maternal transmission of mitochondrial DNA in interspecific hybrids of Populus. Curr. Genet. 22, 141-145 (1992).

41. Fang, Z. F., Zhao, S. D. \& Skvortsov, A. K. In Flora of China Vol. 4 (ed Flora of China Editorial Committee) 139-274 (Science Press and St. Louis: Missouri Botanical Garden Press, 1999).

42. He, C. et al. Clonal reproduction and natural variation of Populus canescens patches. Tree Physiol. 30, 1383-1390 (2010).

43. Taberlet, P., Gielly, L., Pautou, G. \& Bouvet, J. Universal primers for amplification of three non-coding regions of chloroplast DNA. Plant Mol. Biol. 17, 1105-1109 (1991).

44. Tuskan, G. A. et al. The genome of black cottonwood, Populus trichocarpa (Torr. \& Gray). Science 313, 1596-1604 (2006).

45. Dieringer, D. \& Schlötterer, C. MICROSATELLITE ANALYSER (MSA): a platform independent analysis tool for large microsatellite data sets. Mol. Ecol. Notes 3, 167-169 (2003).

46. FSTAT, a program to estimate and test gene diversities and fixation indices (Version 2.9.3) v. Version 2.9.3 (2001)

47. Rousset, F. GENEPOP' 007: a complete re-implementation of the GENEPOP software for Windows and Linux. Mol. Ecol. Resour. 8, 103-106 (2008).

48. Weir, B. S. \& Cockerham, C. C. Estimating F-Statistics for the analysis of population structure. Evolution 38, 1358-1370 (1984).

49. Rousset, F. Equilibrium values of measures of population subdivision for stepwise mutation processes. Genetics 142, 1357-1362 (1996).

50. Excoffier, L., Laval, G. \& Schneider, S. Arlequin (version 3.0): An integrated software package for population genetics data analysis. Evol. Bioinform. 1, 47-50 (2005).

51. Peakall, R. \& Smouse, P. E. GENALEX 6: genetic analysis in Excel. Population genetic software for teaching and research. Mol. Ecol. Notes 6, 288-295 (2006).

52. Falush, D., Stephens, M. \& Pritchard, J. K. Inference of population structure using multilocus genotype data: linked loci and correlated allele frequencies. Genetics 164, 1567-1587 (2003).

53. Pritchard, J. K., Stephens, M. \& Donnelly, P. Inference of population structure using multilocus genotype data. Genetics 155, 945-959 (2000).

54. Evanno, G., Regnaut, S. \& Goudet, J. Detecting the number of clusters of individuals using the software STRUCTURE: a simulation study. Mol. Ecol. 14, 2611-2620 (2005)

55. Anderson, E. C. \& Thompson, E. A. A model-based method for identifying species hybrids using multilocus genetic data. Genetics 160, 1217-1229 (2002).

56. Thompson, J. D., Gibson, T. J., Plewniak, F., Jeanmougin, F. \& Higgins, D. G. The CLUSTAL_X windows interface: flexible strategies for multiple sequence alignment aided by quality analysis tools. Nucleic Acids Res. 25, 4876-4882 (1997).

57. Bandelt, H. J., Forster, P. \& Röhl, A. Median-joining networks for inferring intraspecific phylogenies. Mol. Biol. Evol. 16, 37-48 (1999).

58. Arlequin ver. 3. 1: an integrated software package for population genetics data analysis (Computational and Molecular Population Genetic Lab, Institute of Zoology, Bern, 2006).

59. Basten, C. J. \& Asmussen, M. A. The exact test for cytonuclear disequilibria. Genetics 146, 1165-1171 (1997).

60. Asmussen, M. A. \& Basten, C. J. Sampling theory for cytonuclear disequilibria. Genetics 138, 1351-1363 (1994).

\section{Acknowledgements}

This work was supported by the Fundamental Research Funds for the Central Non-profit Research Institution of CAF (CAFYBB2016ZX001-2) and the Special Fund for Forest Scientific Research in the Public Welfare (201004035). We thank Ai-min Liu and his colleagues from the Flatland Forestry Center of the Haba River in Xinjiang for help with the sampling.

\section{Author Contributions}

Y.-F.Z. conducted molecular laboratory work, analyzed the data, and wrote the paper; Y.-F.Z. and J.-G.Z. conceived the experiments; Y.-F.Z., J.-G.Z., A.-G.D. and B.J. collected the samples.

Additional Information

Supplementary information accompanies this paper at http://www.nature.com/srep

Competing financial interests: The authors declare no competing financial interests.

How to cite this article: Zeng, Y.-F. et al. Genetic structure of Populus hybrid zone along the Irtysh River provides insight into plastid-nuclear incompatibility. Sci. Rep. 6, 28043; doi: 10.1038/srep28043 (2016).

(c) (i) This work is licensed under a Creative Commons Attribution 4.0 International License. The images unless indicated otherwise in the credit line; if the material is not included under the Creative Commons license, users will need to obtain permission from the license holder to reproduce the material. To view a copy of this license, visit http://creativecommons.org/licenses/by/4.0/ 